\title{
Research Progress on the SERPINE1 Protein and Chronic Inflammatory Diseases of the Upper Respiratory Tract: A Literature Review
}

\author{
Teng-yu Chen ${ }^{a}$ Min Zhou ${ }^{b}$ Man-qing Lin ${ }^{a}$ Shu-ting Liang ${ }^{a}$ Yan Yan ${ }^{a}$ \\ Si-min Wanga Cai-shan Fang ${ }^{\text {a }}$ Dan Li $^{\mathrm{a}}$ Yan Ruan ${ }^{\mathrm{b}}$ \\ ${ }^{a}$ The First Clinical College, Guangzhou University of Chinese Medicine, Guangzhou, China; bepartment of \\ Otolaryngology, The First Affiliated Hospital of Guangzhou University of Chinese Medicine, Guangzhou, China
}

\section{Keywords}

SERPINE1 - Upper respiratory diseases · Airway remodelling · Chronic rhinosinusitis - Allergic rhinitis

\begin{abstract}
SERPINE1 protein is one important member of the serine proteinase inhibitor E superfamily that plays a crucial role in the fibrinolytic system. It has been identified which is related to chronic inflammatory lung diseases like allergic asthma and lung fibrosis. Recently, researchers have focused on the impact of SERPINE1 and its genetic polymorphisms on inflammatory diseases of the upper respiratory tract. In this review, we conclude that SERPINE1 is widely involved in the pathological process of chronic rhinosinusitis and allergic rhinitis (AR) and may play a pivotal role in tissue remodelling in chronic rhinosinusitis without nasal polyps. It is also found that the $4 \mathrm{G}$ allele of SERPINE1 gene is associated with the risk of upper respiratory diseases. More studies are needed to further clarify how SERPINE1 influences chronic rhinosinusitis and $A R$, which would be conducive to improving the therapeutic efficacy of treatments for upper respiratory diseases.

(c) 2021 S. Karger AG, Basel
\end{abstract}

\section{Introduction}

The SERPINE1 protein is an important member of the E superfamily of serine proteinase inhibitors. The SERPINE1 protein can quickly inhibit the formation of plasmin [1]. Basing on its effects on fibrinolytic functions, SERPINE1 is involved in chronic inflammation $[2,3]$, tumour metastasis [3, 4], tissue fibrosis [5], and other pathological processes involving the heart [6, 7], lung [8], kidney [9], breast [10], and other organs and has a wide range of biological activities.

In recent years, research on the relationship between pulmonary interstitial fibrosis, bronchial asthma and certain important lower respiratory tract diseases and the SERPINE1 protein has been relatively systematic, and there have been some attempts to explore the application of SERPINE1 inhibitors to treat pulmonary fibrosis and asthma diseases $[11,12]$. The role of SERPINE1 in chronic inflammatory diseases of the upper respiratory tract has also recently received further attention. Our previous study showed that the expression of SERPINE1 in chron-

Edited by: D.Y. Wang, Singapore.

Correspondence to:

Yan Ruan, ruanyan63@163.com 
Fig. 1. The inhibitory effect of SERPINE1 on plasminogen activators under pathological conditions. Normally, the SERPINE1 protein binds t-PA or u-PA, mediating the conversion of plasminogen into active plasmin, which can hydrolyse fibrin into FDPs. Plasmin can also activate MMPs, and both proteins can degrade the extracellular matrix. While in pathological conditions, increasing SERPINE1 further inhibits u-PA or t-PA and indirectly inhibits the proteolytic action of plasmin and MMPs, leading to the deposition of the extracellular matrix, mainly formed as fibrins. SERPINE1, plasminogen activator inhibitor-1; u-PA, urokinase-type plasminogen activator; t-PA, tissue-type plasminogen activator; FDP, fibrin degradation products; MMPs, matrix metalloproteinases.

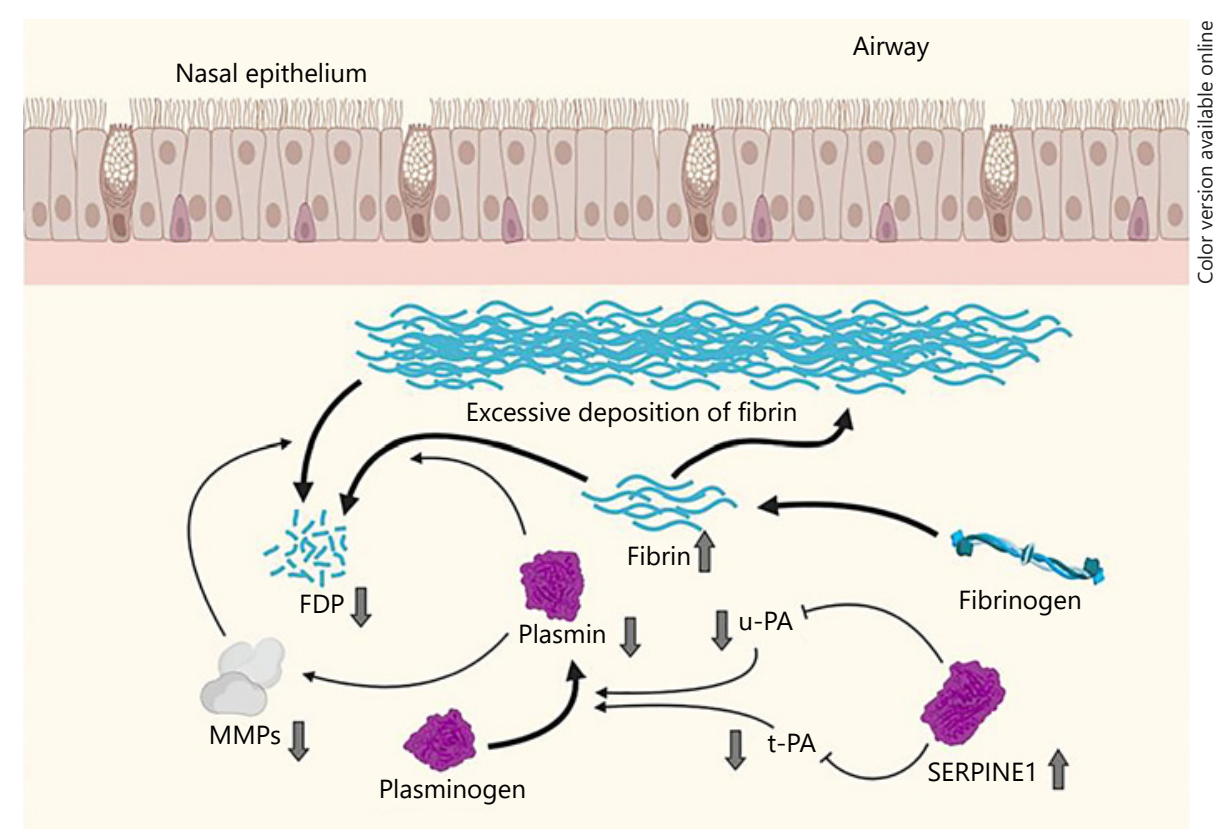

ic sinusitis (CRS) exosomes was higher than that in healthy sinus mucosal tissues [13]. To clearly illustrate the role of SERPINE1 in chronic inflammation in the upper respiratory tract, we will review the relationship between SERPINE1 and common chronic inflammatory diseases of the upper respiratory tract, such as CRS and allergic rhinitis (AR), in the present article.

\section{Basic Features of SERPINE1}

\section{Molecular Structure and Biological Function of SERPINE1}

The SERPINE1 protein is a single-chain glycoprotein composed of 379 amino acids that are mainly synthesized and secreted by platelets, megakaryocytes, hepatocytes, adipocytes, smooth muscle cells, and vascular endothelial cells. The active site of the protein is Arg346Met347. The expression levels of SERPINE1 are associated with the circadian rhythm, with a peak in the morning and a significant decrease at night. Protein expression is positively correlated with age and insulin resistance $[14,15]$. The SERPINE1 protein exists as an activated, lytic, or latent form. The plasma levels of free, activated SERPINE1 are low (approximately 6-80 ng/ $\mathrm{mL}$ ), and this form of the protein is very unstable. The half-life of activated SERPINE1 at $37^{\circ} \mathrm{C}$ is $1-2 \mathrm{~h}$, transforming spontaneously into the latent form. Otherwise, after SERPINE1 binds to the target protease and is cleaved, it irreversibly becomes the cleaved form or substrate form $[1,16,17]$. Due to the instability of the monomeric form, SERPINE1 mostly exists as a complex within the body. SERPINE1 binds to vitronectin, increasing its activity more than 10 -fold and exerting inhibitor-independent effects, and this binding is reversible and does not affect the ability of SERPINE1 to inhibit plasminogen activators $[18,19]$.

Additionally, in the fibrinolytic system, SERPINE1 plays a physiological role as an inhibitor of fibrinogen activators. Urokinase-type plasminogen activator (uPA) could bind reversibly to its specific receptor uPAR to form a u-PA-uPAR complex, and the active peptide bond on the surface of SERPINE1 could irreversibly form a 1:1 complex with the uPA-uPAR complex via a covalent bond. The SERPINE1 protein becomes deactivated after this binding [20]. Tissue-type plasminogen activator ( $\mathrm{t}-\mathrm{PA}$ ) and $\mathrm{u}-\mathrm{PA}$, both of which are plasminogen activators, can mediate the conversion of plasminogen into active plasmin which hydrolyses fibrin into small peptides, such as fibrin degradation products [1]. Plasmin can also activate matrix metalloproteinases (MMPs), and both proteins can degrade the extracellular matrix, while under pathological conditions, abnormally elevated SERPINE1 exerts further inhibitory effects on u-PA and t-PA and indirectly inhibits the proteolytic action of plasmin and MMPs, resulting in the deposition of the extracellular matrix [21,22], as shown in Figure 1. 
SERPINE1 Gene Promoter Polymorphisms in Allergy and Asthma

The human SERPINE1 gene is located on the long arm of chromosome 7 (7q21.3-q22). 4G/5G polymorphism (rs1799762), allele in the SERPINE1 gene promoter region at $-675 \mathrm{bp}$, is a common SERPINE1 gene single nucleotide polymorphism that manifests as a single guanine base deletion or insertion and affects the transcriptional activity of SERPINE1. Usually, there are 5 guanine base sequences around this single-nucleotide variation region, and so, it is often referred to as a $5 \mathrm{G}$ allele, while the $4 \mathrm{G}$ allele manifests a deletion of 1 nucleotide. The existence of the $5 \mathrm{G}$ site of SERPINE1 enables combination with a repressor factor, producing lower levels of SERPINE1 protein [23]. It has been reported that nearly a quarter of people with homozygous for $4 \mathrm{G}$ allele have approximately $30 \%$ higher levels of SERPINE1 proteins than those with homozygous for 5G [24]. According to this feature, the $4 \mathrm{G} / 4 \mathrm{G}$ genotype, $4 \mathrm{G} / 5 \mathrm{G}$ genotype, and $5 \mathrm{G} / 5 \mathrm{G}$ genotype are 3 kinds of SERPINE1 genotypes [25]. Some previous studies have shown that SERPINE1 4G/5G polymorphisms are closely related to asthma airway remodelling. SERPINE1 polymorphisms and early lower respiratory tract infections in the Latino population may correlate with asthma severity and decreased lung function $[26,27]$. A meta-analysis suggested that SERPINE1 gene polymorphisms are significantly related to an increased risk of allergic diseases, which is a risk factor for asthma and is closely related to asthma susceptibility [28, 29].

\section{The Relationship between SERPINE1 and Chronic Inflammation of the Upper Respiratory Tract}

\section{The Relationship between SERPINE1 and CRS}

Microscopic Distribution of Fibrinolytic and

Inhibitory Components in the Mucosa in CRS

Yasuda et al. [30] analysed the fibrinolytic components of the inferior turbinate mucosal tissues and nasal secretions of patients with common nasal diseases during surgical resection or biopsy who had mainly been diagnosed with purulent sinusitis or AR. The immunohistochemical staining results showed that the SERPINE1 protein was mainly distributed in the serous cells of the lamina propria glands. There was no significant difference in the staining of the mucosal epithelium between the patient and normal groups. Moreover, the average level of SERPINE1 in the nasal secretions of patients with purulent sinusitis was higher than that of the normal group.
However, they collected samples from limited patients with different kinds of diseases in one group. Large individual differences might impact on credibility of the results [30]. Sejima et al. [31] further explored the differences in the expression of fibrinolytic components and SERPINE1 in the pathological process of CRS with nasal polyps (CRSwNP) and without nasal polyps (CRSsNP). The researchers collected ethmoid sinus mucosa in CRSsNP, nasal polyp tissue in CRSwNP, and inferior turbinate mucosa from volunteers without sinus disease as a control group. They found that SERPINE1 in the control and CRSwNP group was distributed in glands and fibroblasts, respectively, whereas SERPINE1 in the CRSsNP group was intensively present in glands, epithelium, endothelium, fibroblasts and infiltrated cells, especially infiltrated cells in the submucosal area. The concentration of SERPINE1 in the CRSsNP group was significantly higher than that in the other 2 groups, as determined by ELISA, whereas the ratio of SERPINE1/u-PAR in the CRSwNP group was significantly lower than that in the other groups [31]. This finding suggests that the expression of $\mathrm{u}-\mathrm{PA}$ plays a dominant role in CRSwNP, while SERPINE1 plays a more important role in the pathological process of CRSsNP. In addition, the concentration of transforming growth factor- $\beta 1$ (TGF- $\beta 1$ ) in CRSsNP was positively correlated with SERPINE1 expression. TGF- $\beta 1$ can induce p53 to form a complex with the SERPINE1 gene promoter Smad $2 / 3$ and promote the transcriptional activity of SERPINE1, which was consistent with the results of this study [32]. A study conducted in mainland China showed that SERPINE1 was distributed in the cytoplasm of inflammatory cells in the epithelium, vascular endothelium, glands, and stroma in nasal polyp tissue, which was consistent with the results of the study by Sejima $[31,33]$.

Differences in Fibrinolytic Components and

SERPINE1 Expression in the Various Phenotypes of the CRS Mucosa

Takabayashi et al. [34] further explored the pathological mechanism of fibrin deposition in CRSwNP patients and found that the t-PA level and activity in nasal polyp samples were significantly lower than those in mucosal tissues from the uncinate process in CRS patients and normal controls, which negatively correlated with level of eosinophilic cationic proteins, whereas level of SERPINE1 protein in polyp tissues did not increase. Therefore, the researchers hypothesized that the reason for the decrease or inactivation of $\mathrm{t}-\mathrm{PA}$ in nasal polyps was not SERPINE1 but could be the immune response of Th2 cy- 
tokines, such as IL-4 and IL-13, downregulating t-PA and reducing t-PA expression. In addition, the researchers also measured the fibrinolytic components in the uncinate mucosa and inferior turbinate mucosa of patients and normal volunteers and found that the expression levels of t-PA and u-PA in the uncinate mucosa of patients with both CRSwNP and CRSsNP were significantly lower than those in the inferior turbinate mucosal tissue, whereas there was no significant difference in the t-PA expression levels in the two types of mucosal tissues in the normal group. This finding suggests that studies on SERPINE1 and the two different CRS phenotypes may need to adopt different research strategies in the future [34].

Mueller et al. [35] compared the correlation between CRSwNP and different serine protease inhibitor subfamily members. The researchers selected 4 serpin proteins, SERPINB2, SERPINE1, SERPINF2, and SERPING1, as the research indicators. Western blot results showed that all serpin proteins, including SERPINE1, were overexpressed in the mucosal tissues of patients with CRSwNP. Excessive serpin protein expression caused an imbalance in the proteolytic cascade and downregulated fibrinolytic activity, which may lead to fibrin deposition in nasal mucosal tissue. However, the expression of SERPINE1 in this study was not as significant as that of other serine protease inhibitors. Therefore, the role of other types of serine protease inhibitors in CRSwNP may also warrant further study in the future [35].

\section{SERPINE1 Gene Promoter Polymorphisms in CRS}

As research on the polymorphisms of the SERPINE1 gene promoter have been carried out in multiple disciplines, the characteristics of SERPINE1 gene polymorphisms in upper respiratory diseases have gradually attracted attention. Research on SERPINE1 gene polymorphisms in CRS was subsequently conducted and showed that the frequency of $4 \mathrm{G}$ alleles in the mucosa or polyp tissue from CRS patients without eosinophil infiltration was higher than that of CRS patients with eosinophil infiltration and normal mucosa, but there was no statistically significant difference, suggesting that the $4 \mathrm{G}$ allele of SERPINE1 may correlate with non-eosinophil-infiltrating CRS, but more studies are still needed to verify this hypothesis [36].

\section{The Relationship between SERPINE1 and AR}

SERPINE1 in Fibrinolytic System in AR

Sejima not only focused on the role of the SERPINE1 protein in CRS but also studied the characteristics of the fibrinolytic system in AR patients in the early stage and found that the mRNA expression of $\mathrm{u}-\mathrm{PA}$ and SERPINE1 in the nasal mucosa of AR patients was significantly higher than that in normal human nasal mucosa. SERPINE1 mRNA can be detected in serous cells and the epithelium. The immunohistochemical results showed that SERPINE1 was mainly distributed in the serous cells of AR submucosal glands, while the epithelium was hardly stained [37]. Another study showed that the mRNA expression levels of coagulation system components such as coagulation factor II and coagulation factor $\mathrm{X}$ were significantly increased in AR mice. Although the expression of SERPINE1 mRNA showed an increasing trend, there was no significant difference compared with that of control mice. u-PA mRNA expression was significantly downregulated in AR group mice, suggesting that the difference in the composition of the coagulation and fibrinolytic systems may be related to mucosal fibrin deposition in mice [38].

\section{SERPINE1 and Immune Response in AR}

To further clarify the relationship between SERPINE1 and the type of immune response in AR patients, Sejima et al. [39] carried out another animal experiment and found that the level of active SERPINE1 detected in the nasal lavage fluid of SERPINE1-knockout (SERPINE1 ${ }^{-/-}$) OVA-sensitized mice was lower than that of wild-type OVA-sensitized mice in the research. Interestingly, reduced IL-4 and IL-5 levels as well as increased IFN- $\gamma$ levels were detected in SERPINE1 ${ }^{-/-}$mice. In addition, mucous cells such as goblet cells in the nasal septum and infiltrated eosinophils were significantly increased in wild-type mice, while only a few neutrophils could be detected in SERPINE1 ${ }^{-1-}$ mice. IL-4 and IL-5 are important cytokines in Th2 immune response, while IFN $-\gamma$ is one of the most crucial cytokines in Th1 immune response. The study suggested that SERPINE1 might be closely related to Th2 immune response and the lack of SERPINE1 appeared to impact on immunotype in AR. In another study, the t-PA gene was knocked out in AR model mice, and the expression of the $\mathrm{u}-\mathrm{PA}$ and SERPINE1 genes increased significantly in both t-PA knockout mice and wild-type mice sensitized by OVA, while the expression of MMP-9 was reduced. The collagen deposition in AR model mice in the knockout group was significantly more severe than that in the wild-type group, suggesting that the $\mathrm{t}$-PA-induced extracellular matrix deposition in the nasal mucosa may not be directly related to u-PA or SERPINE1 [40]. Moreover, some studies have preliminarily examined whether fibrinolytic components differ in intermittent and persistent AR and showed no significant 
differences in serum u-PA, uPAR, and SERPINE1 levels between the two kinds of rhinitis, but some factors may have led to negative outcomes in this study, such as the limited sample size and low average age of the selected patients. In addition, the patients included in the study were limited to those who had a positive reaction to grass pollen or house dust mites, which may be one of the possible factors contributing to bias. More large-sample clinical trials are needed for further corroboration [41].

SERPINE1 Gene Promoter Polymorphisms in AR

SERPINE1 gene polymorphisms in AR patients have also been examined by some scholars. A Turkish study showed that SERPINE1 $4 \mathrm{G}$ alleles were common in AR children and the lung function of children with the $5 \mathrm{G} / 5 \mathrm{G}$ genotype was significantly better than those with the $4 \mathrm{G} / 5 \mathrm{G}$ or $4 \mathrm{G} / 4 \mathrm{G}$ genotype. There is little evidence that SERPINE1 gene polymorphisms are related to the total serum IgE level, total eosinophil count, or positive skin prick test outcomes in children with AR [42]. In addition, in people with asthma, patients with AR symptoms carry the SERPINE1 4G/4G genotype more frequently (28.1\%), and asthma patients with at least one $4 \mathrm{G}$ allele have an increased risk of developing AR symptoms [43]. However, in recent years, few studies have focused on the 4G/5G SERPINE1 polymorphism in AR patients. Additional supporting studies are needed to further explore the relationship between different populations or subtypes of AR and gene polymorphisms.

\section{Conclusion}

According to current studies on chronic inflammation of the upper respiratory tract, SERPINE1 is mainly involved in the pathological processes of CRS and AR. SERPINE1 may play a more important role in the pathological process of CRSsNP than in CRSwNP, such as in tissue remodelling. The roles of the SERPINE1 protein and gene polymorphisms in CRS and AR have been preliminarily explored, and there is a certain correlation between $4 \mathrm{G}$ alleles and the risks of diseases. Future research should focus of the SERPINE1 protein to explore its roles in the pathological processes of different CRS phenotypes and the way in which SERPINE1 participates in the immune response in upper respiratory tract inflammatory diseases. The relationship between SERPINE1 gene polymorphisms in AR and asthma, as well as their different characteristics, require further research. Whether the SERPINE1 protein is suitable for evaluating the severity of CRS, especially tissue fibrosis in CRSsNP, and whether it is suitable for the clinical evaluation of the efficacy and prognosis of CRS medications is also a research direction that deserves attention. In-depth research on the mechanism of chronic inflammatory diseases of the upper respiratory tract would be beneficial to understanding the common pathological mechanisms of upper and lower respiratory tract diseases and further enrich the treatment of the respiratory system diseases.

\section{Conflict of Interest Statement}

The authors have no relevant conflicts of interest to declare.

\section{Funding Sources}

The study was supported by General Project of National Science Foundation of China (Grant No. 81974581).

\section{Author Contributions}

Teng-yu Chen and Min Zhou conceptualized the main idea of this review and drafted most of the sections of the manuscript. They contributed equally to this work and should be considered as co-first authors. Man-qing Lin and Shu-ting Liang mainly completed the writing of "SERPINE1 Gene Promoter Polymorphisms in CRS" and "SERPINE1 Gene Promoter Polymorphisms in AR." Yan Yan completed the writing of the conclusion. Si-min Wang, Cai-shan Fang, and Dan Li mainly retrieved the information from databases, collected relevant references, and supplemented the content of the article. Yan Ruan critically revised the whole draft of the manuscript and provided details of authorship and disclosures of relationships.

\section{References}

1 Yin Y. Review on the researches of the functions of fibrinolytic system. J Southern Med Univ. 2004;(2):191-4. Chinese.

2 Tiwari N, Marudamuthu AS, Tsukasaki Y, Ikebe $\mathrm{M}, \mathrm{Fu}$ J, Shetty S. p53- and PAI-1-mediated induction of C-X-C chemokines and CXCR2: importance in pulmonary inflammation due to cigarette smoke exposure. Am J Physiol Lung Cell Mol Physiol. 2016 Mar 15;310(6):L496-506.

3 Dong W, Wu X. Overexpression of Rab11FIP2 in colorectal cancer cells promotes tumor migration and angiogenesis through increasing secretion of PAI-1. Cancer Cell Int. 2018;18(1):35.
4 Wei X, Li S, He J, Du H, Liu Y, Yu W, et al. Tumor-secreted PAI-1 promotes breast cancer metastasis via the induction of adipocytederived collagen remodeling. Cell Commun Signal. 2019;17(1):58.

5 Ghosh AK, Vaughan DE. PAI-1 in tissue fibrosis. J Cell Physiol. 2012;227(2):493-507. 
6 Zaman AK, French CJ, Schneider DJ, Sobel BE. A profibrotic effect of plasminogen activator inhibitor type-1 (PAI-1) in the heart. Exp Biol Med. 2009;234(3):246-54.

$7 \mathrm{Xu} \mathrm{Z}$, Castellino FJ, Ploplis VA. Plasminogen activator inhibitor-1 (PAI-1) is cardioprotective in mice by maintaining microvascular integrity and cardiac architecture. Blood. 2010 Mar 11;115(10):2038-47.

8 Wang H, Yang T, Li D, Wu Y, Zhang X, Pang $\mathrm{C}$, et al. Elevated circulating PAI-1 levels are related to lung function decline, systemic inflammation, and small airway obstruction in chronic obstructive pulmonary disease. Int $J$ Chron Obstruct Pulmon Dis. 2016 Jan 20;11: 2369-76.

9 Huang Y, Border WA, Lawrence DA, Noble NA. Noninhibitory PAI-1 enhances plasminmediated matrix degradation both in vitro and in experimental nephritis. Kidney Int. 2006 Jan 1;70(3):515-22.

10 Andres SA, Edwards AB, Wittliff JL. Expression of urokinase-type plasminogen activator (uPA), its receptor (uPAR), and inhibitor (PAI-1) in human breast carcinomas and their clinical relevance. J Clin Lab Anal. 2012 Feb 1;26(2):93-103.

11 Huang WT, Vayalil PK, Miyata T, Hagood J, Liu RM. Therapeutic value of small molecule inhibitor to plasminogen activator inhibitor-1 for lung fibrosis. Am J Respir Cell Mol Biol. 2012 Jan 1;46(1):87-95.

12 Lee SH, Eren M, Vaughan DE, Schleimer RP, Cho $\mathrm{SH}$. A plasminogen activator inhibitor-1 inhibitor reduces airway remodeling in a murine model of chronic asthma. Am J Respir Cell Mol Biol. 2012;46(6):842-6.

13 Zhou M, Tan KS, Guan WJ, Jiang LJ, Deng J, Gao WX, et al. Proteomics profiling of epithelium-derived exosomes from nasal polyps revealed signaling functions affecting cellular proliferation. Respir Med. 2020;162:105871.

14 Huber K, Rosc D, Resch I, Schuster E, Glogar DH, Kaindl F, et al. Circadian fluctuations of plasminogen activator inhibitor and tissue plasminogen activator levels in plasma of patients with unstable coronary artery disease and acute myocardial infarction. Thromb Haemost. 1988 Dec 22;60(3):372-6.

15 Sobel BE, Lee YH, Pratley RE, Schneider DJ. Increased plasminogen activator inhibitor type-1 (PAI-1) in the heart as a function of age. Life Sci. 2006;79(17):1600-5.

16 Liu R-M. Oxidative stress, plasminogen activator inhibitor 1, and lung fibrosis. Antioxid Redox Signal. 2008;10(2):303-20.

17 Lindahl TL, Sigurdardottir O, Wiman B. Stability of plasminogen activator inhibitor 1 (PAI-1). Thromb Haemost. 1989 Sep 29; 62(2):748-51.

18 Salonen EM, Vaheri A, Pöllänen J, Stephens $\mathrm{R}$, Andreasen P, Mayer M, et al. Interaction of plasminogen activator inhibitor (PAI-1) with vitronectin. J Biol Chem. 1989 Apr 15; 264(11):6339-43.
19 Zhou A, Huntington JA, Pannu NS, Carrell RW, Read RJ. How vitronectin binds PAI-1 to modulate fibrinolysis and cell migration. Nat Struct Biol. 2003 Jul 1;10(7):541-4.

20 Planus E, Barlovatz-Meimon G, Rogers RA, Bonavaud S, Ingber DE, Wang N. Binding of urokinase to plasminogen activator inhibitor type-1 mediates cell adhesion and spreading. J Cell Sci. 1997 Jan 1;110(Pt 9):1091.

21 Lijnen HR. Plasmin and matrix metalloproteinases in vascular remodeling. Thromb Haemost. 2001 Jul 1;86(1):324-33.

22 Janssens S, Lijnen HR. What has been learned about the cardiovascular effects of matrix metalloproteinases from mouse models? Cardiovasc Res. 2006 Feb 15;69(3):585-94.

23 Grant PJ. Polymorphisms of coagulation/fibrinolysis genes: gene environment interactions and vascular risk. Prostaglandins Leukot Essent Fatty Acids. 1997 Jan 1;57(4-5): 473-7.

24 Humphries SE, Panahloo A, Montgomery HE, Green F, Yudkin J. Gene-environment interaction in the determination of levels of haemostatic variables involved in thrombosis and fibrinolysis. Thromb Haemost. 1997 Jan 1;78(1):457-61.

25 Zorio E, Gilabert-Estellés J, España F, Ramón LA, Cosín R, Estellés A. Fibrinolysis: the key to new pathogenetic mechanisms. Curr Med Chem. 2008 Jan 1;15(9):923-9.

26 Zhang X, Lin J, Yang M, He J, Huang X, Cai $\mathrm{Z}$, et al. Role of $4 \mathrm{G} / 5 \mathrm{G}$ polymorphism of PAI1 gene in airway remodeling. J Clin Med Practice. 2009;13(23):1-6. Chinese.

27 Cho SH, Min JY, Kim DY, Oh SS, Torgerson DR, Pino-Yanes M, et al. Association of a PAI-1 gene polymorphism and early life infections with asthma risk, exacerbations, and reduced lung function. PLoS One. 2016 Aug 24;11(8): 0157848.

28 Nie W, Li B, Xiu QY. The -675 4G/5G polymorphism in plasminogen activator inhibitor-1 gene is associated with risk of asthma: a meta-analysis. PLoS One. 2012 Jan 20;7(3): e34385.

29 Zheng L, Li X, Song Q, Hou C, Chen X, Li B. PAI-1 gene polymorphism was associated with an increased risk of allergic diseases: evidence from a meta-analysis of 14 case-control studies. Int Arch Allergy Immunol. 2019; 180(4):255-63.

30 Yasuda T, Sakata Y, Madoiwa S, Mimuro J, Matsuda M, Kitamura K. Fibrinolytic components in nasal mucosa and nasal secretion. Histochem Cell Biol. 1998 Jan 1;110(5):44955.

31 Sejima T, Holtappels G, Bachert C. The expression of fibrinolytic components in chronic paranasal sinus disease. Am J Rhinol Allergy. 2011 Jan 1;25(1):1-6.
32 Kawarada Y, Inoue Y, Kawasaki F, Fukuura K, Sato K, Tanaka T, et al. TGF- $\beta$ induces p53/ Smads complex formation in the PAI- 1 promoter to activate transcription. Sci Rep. 2016; 6(1):35483.

$33 \mathrm{Hu}$ Y. Expression and clinical significance of MMP-7 TGF- $\beta 1$ PAI-1 in nasal polyps. Zhengzhou, China: Zhengzhou University; 2010. Chinese.

34 Takabayashi T, Kato A, Peters AT, Hulse KE, Suh LA, Carter R, et al. Excessive fibrin deposition in nasal polyps caused by fibrinolytic impairment through reduction of tissue plasminogen activator expression. Am J Respir Crit Care Med. 2013 Jan 1;187(1):49-57.

35 Mueller SK, Nocera AL, Dillon ST, Libermann TA, Wendler O, Bleier BS. Tissue and exosomal serine protease inhibitors are significantly overexpressed in chronic rhinosinusitis with nasal polyps. Am J Rhinol Allergy. 2019 Jan 1;33(4):359.

36 de Alarcon A, Steinke JW, Caughey R, Barekzi E, Hise K, Gross CW, et al. Expression of leukotriene $\mathrm{C} 4$ synthase and plasminogen activator inhibitor 1 gene promoter polymorphisms in sinusitis. Am J Rhinol. 2006 Sep 1; 20(5):545-9.

37 Sejima T, Madoiwa S, Mimuro J, Sugo T, Ishida T, Ichimura K, et al. Expression profiles of fibrinolytic components in nasal mucosa. Histochem Cell Biol. 2004 Jan 1;122(1):6173.

38 Hong SN, Zhang YL, Rhee CS, Kim DY. Probable roles of coagulation cascade and fibrinolysis system in the development of allergic rhinitis. Am J Rhinol Allergy. 2019;33(2):13744.

39 Sejima T, Madoiwa S, Mimuro J, Sugo T, Okada K, Ueshima S, et al. Protection of plasminogen activator inhibitor-1-deficient mice from nasal allergy. J Immunol. 2005 Jan 1; 174(12):8135-43.

40 Hua H, Zhang R, Yu S, Wang H, Zhao Y, Yu $\mathrm{S}$, et al. Tissue-type plasminogen activator depletion affects the nasal mucosa matrix reconstruction in allergic rhinitis mice. Allergol Immunopathol. 2010;39(4):206-11.

41 Kasperska-Zajac A, Brzoza Z, Rogala B. The urokinase system in patients with intermittent and persistent allergic rhinitis. Blood Coagul Fibrinolysis. 2008 Jan 1;19(7):685-8.

42 Ozbek OY, Ataç FB, Ogus E, Ozbek N. Plasminogen activator inhibitor-1 gene 4G/5G polymorphism in Turkish children with asthma and allergic rhinitis. Allergy Asthma Proc. 2009 Jan 1;30(1):41-6.

43 Lampalo M, Jukic I, Bingulac-Popovic J, Marunica I, Petlevski R, Pavlisa G, et al. Polymorphism $4 \mathrm{G} / 5 \mathrm{G}$ of the plasminogen activator inhibitor 1 gene as a risk factor for the development of allergic rhinitis symptoms in patients with asthma. Eur Arch Otorhinolaryngol. 2017 Jan 1;274(6):2613-9. 